\title{
Plane Grating Monochromators for Synchrotron Radiation*
}

by

\author{
Malcolm R. Howells
}

The National Synchrotron Light Source

Brookhaven National Laboratory, Upton, NY 11973

\section{ABSTRACT}

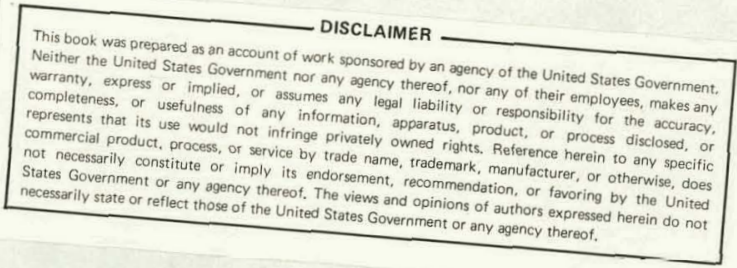

The general background and theoretical basis of plane grating monochromators (PGM's) is reviewed and the particular case of grazing incidence PGM's suitable for use with synchrotron radiation is considered in detail. The theory of reflection filtering is described and the problem of the finite source distance is shown to be of special importance with high brightness storage rings. The design philosophy of previous instruments is discussed and a new scheme proposed, aimed at dealing with the problem of the finite source distance. This scheme, involving a parabolic collimating mirror fabricated by diamond turning, is considered in the context of Wolter-type telescopes and microscopes. Some practical details concerning an instrument presently under construction using the new design are presented.

DHSTRIBUTION OF FRIS OOCUMENT IS UNLIMITEB

*Research supported by the U.S. Department of Energy. 


\section{DISCLAIMER}

This report was prepared as an account of work sponsored by an agency of the United States Government. Neither the United States Government nor any agency Thereof, nor any of their employees, makes any warranty, express or implied, or assumes any legal liability or responsibility for the accuracy, completeness, or usefulness of any information, apparatus, product, or process disclosed, or represents that its use would not infringe privately owned rights. Reference herein to any specific commercial product, process, or service by trade name, trademark, manufacturer, or otherwise does not necessarily constitute or imply its endorsement, recommendation, or favoring by the United States Government or any agency thereof. The views and opinions of authors expressed herein do not necessarily state or reflect those of the United States Government or any agency thereof. 


\section{DISCLAIMER}

Portions of this document may be illegible in electronic image products. Images are produced from the best available original document. 
The use of plane gratings for spectroscopy dates from around 1882 when Rowland 1,2 developed the technique of ruling to a sufficiently refined level. At about the same time he discovered the famous focussing property of the concave grating ${ }^{3}$. The two types have thus developed side by side and have competed for the favor of instrument designers.

Generally speaking, plane gratings are cheaper to produce so they are preferred where their use is feasible. This has generally been in the range where lens collimators can be used $(\lambda>1600 \AA)$ with typically a Littrow spectrograph 4 configuration. Alternatively, for a monochromator, Ebert-Fastie 5 , or Czerny-Turner ${ }^{6}$ arrangements have been popular. These work best in the range $(\lambda>1000 \AA)$ where efficient normal incidence reflectors are available. For wavelengths below $1000 \AA$, concave grating spectrometers have generally been preferred.

Concave gratings have the great advantage that collimation, dispersion and focussing are all effectively carried out by one device with only one reflection. Since reflectors below $1000 \AA$ are generally not very efficient, this is a great advantage. Even below $300 \AA$ where grazing angles of incidence must be used the concave grating still retains advantages. It is true it suffers from severe astigmatism (i.e., it focusses well, only in one plane) but in this range additional problems arise in using plane gratings. These concern the provision of collimating and focussing optics. Not only are the extra reflectors inefficient (degrading through-put), but suffer from serious aberrations (tending to degrade resolution). The aberrations can be reduced (but not eliminated) by use of aspheric reflectors. These in turn are expensive and difficult to produce and such replication methods as are presently available tend to be incompatible with the ultra high vacuum environment that frequently is involved in modern spectroscopy in the 10-1000 range. Grazing incidence spectrographs using the concave grating are thus fairly popular, but for monochromators there can be special problems. 
Sowe discussion of the issues involved in addressing these probiems in the use of the concave grating with particular reference to grazing incidence monochromators has already been offered by the author ${ }^{7}$. This discussion concerned the special case of a synchrotron light source. In this case, the light source has considerable natural collimation and this, together with certain other considerations discussed in reference 7 , has prompted various designers to construct plane grating monochromators (PGM's) for use with synchrotron radiation. It is always taken as a constraint that both the synchrotron source and experiment are immovable so that the monochromator must have constant, preferably zero, deviation. Here we confine our attention to instruments that satisfy that condition.

Since the number of synchrotron light sources existing, under construction or planned seems to increase annually, it appears opportune to review the design questions that arise and the contributions that can be expected from plane grating devices to this branch of spectroscopy. 
Summarizing the discussion in reference 7 , we may list the main characteristics of real instruments that employ the kind of plane grating approach which we are considering:

(i) Wavelength range can be about 10-1000A.

(ii) The source is effectively the entrance slit so that its size and distance from the instrument determine the best achievable resolution.

(iii) Typically only three reflections are needed.

(iv) Constant deviation is possible.

(v) Reflection filtering is possihle.

(vi) Excellent polarization is normal.

(vii) A simple mechanism (a sine bar) is used for wavelength scan.

(viii) The wavelength scale is linear.

(ix) The light gathering power (étendue) is high because of the large aperture. It is clear from the list that this class of instruments can offer a large fraction of the degired characteristics. Indeed, when used with a high brightness storage ring, both the resolution and etendue are considerably better than any existing Rowl and circle instruments in the difficult 10-100A region. The only important quantity which is compromised is throughput compared to, say, a toroidal grating monochromator. But the compromise is not serious (see later). Good science has already been done with presently existing instruments. And for the future we should note that the modern dedicated synchrotron light sources have much higher brightness than earlier ones and so it is on these that PGM's are expected to get the best resolution and, in this author's opinion, come into their own as the single best general purpose instrument in the 10-1000\& range.

All of the existing designs operate on the general principal shown in fig. 1. Light from the source is incident directly on the grating. Normally, r is large so that the light is approximately collimated. It is diffracted by the grating as shown and then focussed by a mirror onto an exit slit. 


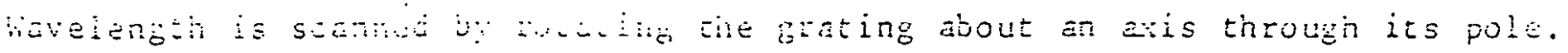

The mirror remains fixed during scanning as do the ingoing and outgoing directions at the grating. For different angles of the grating, different wavelengths are diffracted in the correct direction for being focussed at the exit slit. Using the notation of fig. 2 and the sign convention of Samson 8 , for example, we can state this as:

$$
\begin{aligned}
& \alpha-\beta=2 \theta \\
& \operatorname{Nk} \lambda=\sin \alpha+\sin \beta
\end{aligned}
$$

Where $k$ is the order. Obviously, we can solve (1) and (2) for $x$ and $B$ fnr any $\lambda$ if we first choose $\mathrm{N}$ and $\theta$.

$$
\begin{aligned}
& \beta=\sin ^{-1}\left[\frac{N k \lambda}{2 \cos \theta}\right]-\theta \\
& \alpha=\sin ^{-1}\left[\frac{N k}{2 \cos \theta}\right]+\theta
\end{aligned}
$$

Another effect of working with constant deviation is that there is a limit to the wavelength scan at some 'horizon' wavelength $\lambda_{\mathrm{H}}$ for which $\alpha$ or $B=90^{\circ}$. This is given by:

$$
\lambda_{H}=\frac{2 \cos ^{2} \theta}{N_{k}}
$$

We can derive another expression of rather general application by considering the grating equation as applied to the principle ray at given $\alpha$ and $B$ in fig. 1. If we consider a neighboring ray with angular difference $\Delta \alpha$ coming from a point near $S$ on an extended source, then we have $N k \Delta \lambda=\cos \alpha \Delta \alpha$. We thus see that the effect of a source of finite size(s) is to introduce a source size limit $\Delta \lambda_{s}$ to the resolution given by:

$$
\Delta \lambda_{s}=\frac{\cos \alpha s}{N k r}
$$


:it dipession at the exit slit is given by:

$$
\frac{d}{d q}=\frac{\cos Q}{N h L}
$$

Where $q$ is a lateral co-ordinate in the $p l$ ane of the slit and $L$ is the mirror slit distance.

One can easily compute the light gathering power of the instrument. Suppose we have a grating with rulings of length $\ell$ across a width $\omega$. The Étendue, E (light collection area $x$ solid angle) is given by:

$$
E_{\mathrm{PGM}}=\left[\frac{\omega \ell \cos \alpha}{\mathrm{r}}\right]^{2}
$$

For comparison purposes we give the equivalent relation for a Rowland Circle instrument. In this case, we can speak of well defined $f$ numbers, say $f_{n}^{t}$ in the principal plane and $\frac{\mathrm{fs}}{\mathrm{n}}$ in the plane perpendicular to that. If the entrance slit has width $x$ and length $h$, then

$$
E_{R C}=\frac{h x}{f_{n}^{t} f_{n}^{s}}
$$

For the PGM case the 'entrance slit' is the source and is always the same size. Consequently, $\mathrm{E}$ is independant of the band pass setting, $\Delta \lambda$. For the Rowl and case $E$ depends on $\Delta \lambda$ via the slit width $x$. We can see from this that ( 9 ) and (10) provide the proper way to compare the light gathering power of instruments provided $\mathbf{x}$ is chosen so that they have equivalent resolution.

\section{REFLECTION FILTERING OF HIGHER DIFFRACTED ORDERS}

One of the prime advantages of PGM's is that it is possible to include in the design a provision for filtering out diffracted orders higher than the first. Miyake 9 gave the prescription for doing this and it is of particular importance for certain solid state physics experiments. It is especially necessary with synchrotron sources since the source spectrum usually contains considerably more second order light than first. The method depends on the fact that the reflecting efficiency of materials at wavelengths below about. $500 \AA$ decreases with decreasing wavelength and increasing grazing angle. Thus, an angle of incidence can always be found that gives high efficiency for first order light and low efficiency for higher orders. A particular choice of angle will only be appropriate over a limited wavelength range so we arrive at the 


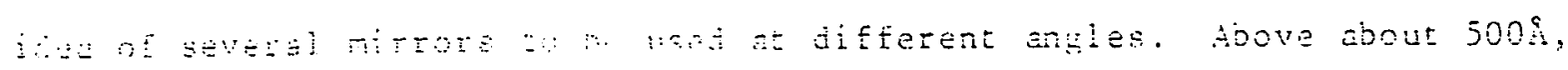

the whoie proces tends to ureul down because of structure in the optical

constants.

In order to choose the correct angles, we follow the argument set out first by Miyake 9 and elaborated by West, et allo. Consider the case of a light wave encountering a diffraction grating coated with material of refractive index $n$ and consider the refracted wave progressing into the material wich angle of refraction $\gamma$. The optical path condition that must be satisfied for the direction $\gamma$ to be a diffraction maximum is:

$$
\text { Nki }=\sin \alpha-n \sin \gamma
$$

To determine the cut-off wavelength, we are interested in the case $\gamma=90^{\circ}$ or if there is a blaze angle $\theta_{B}$ then $\gamma=90^{\circ}+\theta_{B}$. This leads to:

$$
\operatorname{Nk} \lambda=\sin \alpha-n \cos \theta_{B}
$$

For reasons that will become clear later, we will work with $k=-1$. We also use the approximation 12 .

$$
\mathrm{n}=1-\delta \lambda^{2}
$$

where

$$
\delta=\frac{\mathrm{N}^{\rho N} \text { off } \mathrm{e}^{2}}{2 m e^{\pi c^{2} A}}
$$

$N_{0}$ is Avagadro's number, $p$ the density, Neff the number of 'effectively free' electrons/atom e the electronic charge, $m_{c}$ the electronir mass, $c$ tho velocity of light and $A$ the atomic weight. (11) and (12) then give:

$$
\lambda_{1}^{G}=\frac{\left[1+\frac{4 \delta \cos \theta_{B}}{N}\left(\cos \theta_{B}-\sin \alpha\right)\right]^{\frac{1}{2}}-1}{2 \delta \cos \theta_{B / N}}
$$

This gives the threshold wavelength which just begins to be diffracted at $\alpha$. Using (11) and (12) again with $\lambda \rightarrow \lambda / 2$ and $k=-2$, we get the first order wavelength $\lambda_{2}^{G}$ at which second order just begins to be present

$$
\lambda_{2}^{G}=\frac{\left[1+\frac{\delta \cos \theta_{B}}{N}\left(\cos \theta_{B}-\sin \alpha\right)\right]^{\frac{1}{2}}-1}{\delta \cos \theta_{B / 2 N}}
$$


In the imit as $N \rightarrow \infty$ (13) and (14) appiy to a mirror and we get:

$$
\begin{aligned}
& \lambda_{1}^{M}=\left[\frac{1-\sin \alpha}{\delta}\right]^{\frac{1}{2}} \\
& \lambda_{2}^{M}=2\left[\frac{1-\sin \alpha}{\delta}\right]^{\frac{1}{2}}
\end{aligned}
$$

An obvious extension enables the third order thresholds to be included. The best tabulations of $\mathrm{N}_{\mathrm{eff}}$ are those of Hageman, et al 13. Some additional information is collected by Williams and Howells 14 .

Fig. 3 shows plots of $\lambda_{1}^{G} \lambda_{2}^{G} \quad \lambda_{3}^{G} \quad \lambda_{1}^{M} \quad \lambda_{2}^{M}$ and $\lambda_{3}^{M}$ for gold using the data in Table 6 of ref. 13. The grating plots apply to a $600 \mathrm{l} / \mathrm{mm}$ grating. The dotted lines represent the relationship between $\lambda$ and $\alpha$ as the grating is rotated using the $\theta$ values shown. We note various features from this plot.

(i) We see how to cover the wavelength range and achieve the best. possible reflection filtering of second order. We must follow wavelength scan paths which lie between the $\lambda_{1}^{M}$ and $\lambda_{2}^{M}$ curves. For example, one might choose:

$$
\begin{aligned}
10-35 \AA & \theta=88^{\circ} \\
30-90 \AA & \theta=86^{\circ} \\
75-160 \AA & \theta=80^{\circ} \\
150-350 \AA & \theta=67^{\circ}
\end{aligned}
$$

and, therefore, use four mirrors. If second order were absent, as it might be, for other reasons, then filtering out third order would be similar.

(ii) We can see why operating in negative order ( $\alpha$ increasing as $\lambda$ increases) is desirable for higher order suppression. 
(iii) One can see oualitatively what would happen for $\mathrm{N}$ values other than $600 \mathrm{~d} / \mathrm{mm}$. Coarser gratings are more similar to a mirror so that $300 \mathrm{l} / \mathrm{mm}$ grating curves would be closer to the mirror curves, $1200 \mathrm{l} / \mathrm{mm}$ ones further away.

Using the arguments in this section, we can choose a value or values for $\theta$. It is also clear from fig. 3 that good order sorting (at low wavelengths) is easier with a $600 \mathrm{l} / \mathrm{m}$ grating than a $1200 \mathrm{l} / \mathrm{mm}$. In fact, for very grazing applications aimed at the one kilovolt region, we can hardly use a $1200 \mathrm{~h} / \mathrm{mm}$ grating because by (6) if we had $\theta=88^{\circ}$, then $\lambda_{H}$ would be about $20 \AA$. This would be a severe limitation. At longer wavelengths when slightly smaller $\theta$ values are permissible, the $1200 \ell /$ m grating could be used, but the improved resolution it would give is not so important. $600 \mathrm{l} / \mathrm{mm}$ is thus a good working groove denstey for the high entrgy region.

\section{OPTICAL DESIGN OF EXISTING PGM'S}

Turning to the focussing mirror in fig. 1 we encounter a number of possibilities. The simplest is a spherical mirror. Whether the light incident on the mirror from the grating is exactly or approximately parallel, there will always be considerable aberrations. Astigmatism will produce a line image at the exit slit and spherical aberration and coma will broaden and curve the line causing a serious degradation of resolution which can only be controlled by reducing the mirror aperture. While the overall effect is a considerable loss of flux, there is an advantage to the spherical mirror. We can see from the geometry of fig. 1 (using the expression $\frac{1}{2} R \cos \theta$ for the tangential focal length of the mirror) that

$$
y=\frac{1}{2} R \cos \theta-x \cot 2 \theta
$$

For, given $x, y$ and $R$, it turns out that this equation yields two solutions for $\theta$. One can thus use one mirror for two $\theta$ settings, as did Miyake who first employed the method, or, two mirrors for four settings (West, et allo and Howells, et al11). This approach is economical on mirrors but expensive on mechanisms. 
An alternative approach is to use a single fixed, focussing mirror of very grazing angle (i.e., not doing any filtering) and use some other method for filtering. This is the approach adopted by the Hamburg group. They were able to obtain a good quality off-axis paraboloid which, having zero spherical aberration, was able to give an image at its focus with negligible degradation. Their first scheme to achieve filtering of higher orders incorporated an ingenious, double rhombus, mechanism which rotated the grating and rotated and suitably translated a plane pre-mirror. The arrangement had the important extra benefit of maintaining the grating always on blaze. From an optical point of view this scheme, due to Dietrich and Runz15, is probably the most elegant yet produced, however, from a practical point of view it proved difficult to implement. The tolerances involved were severe and considerable time was required for successful alignment and commissioning. For their next instrument, the Hamburg group chose a simplified version of the Dietrich-Kunz design which addressed the modern need for ultra high vacuum compatibility. The result was the so-called 'Flipper'16. This instrument uses a pre-mirror which remains stationary during scanning but which can be selected from a bank of 6 mirrors with a range of different $\theta$ values and surface coatings. This gives a simple scanning mechanism but the mirror interchange still involves some difficult tolerances. This Flipper monochromator has now been operational for about 3 years using $0.75 \mathrm{mr}$ of radiation from the DORIS storage ring. It has achieved the best resolution of the existing PGM's and has operated successfully with a UHV experimental chamber.

One can see that the history of grazing incidence PGM's is quite short and we tabulate the salient features in Table $I$. We also include information about two other instruments which are planned or under construction. Before we can discuss the philosophy of these schemes, we must consider some further aspects of the optical system of fig. 1 . 


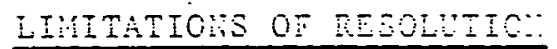

The use of PGM's has historically been associated either with collimated light or very distant sources. For the synchrotron radiation applications on DESY, NINA and DORIS, the source was about 40 m away. This was sufficiently far that even with source sizes of around $2 \mathrm{~mm}$, one could still get source size limited resolutions (eqn 7) that were reasonably actractive. We must now consider the optical effect of the finite source distance on the imaging system to see what is required for the source size limit to be realized.

Various authors have studied the plane grating in non-parallel light 17, 18, 19, For example, Murtyl7 used the standard theory of the spherical grating 20,21 with the radius equal to infinity. Most of the aberration terms are still non-zero and in particualr the focussing term of the spherical grating:

$$
C_{20}=\frac{1 / 2}{2}\left[\left(\frac{\cos ^{2} \alpha}{r}-\frac{\cos \alpha}{R}\right)+\left(\frac{\cos ^{2} \beta}{r^{\prime}}-\frac{\cos \beta}{R}\right)\right]
$$

gives us the focal property of the plane grating. Putting $R=\infty$ and $c_{20}=0$, we find:

$$
\mathbf{r}^{\prime}=-\mathbf{r}\left[\frac{\cos \beta}{\cos \alpha}\right]^{2}
$$

implying that there is a virtual image I distant $r$ 'behind the grating. This image has all the aberrations including astigmatism, coma and spherical aberration, that we would expect for the non-Rowl and spherical grating case. I acts as a virtual object for the focussing mirror to image on to the exit slit. Using the aberration expansion terms and their geometrical optics interpretation 7 , one can show that the image degradation due to aberrations is not normally serious. The major effect is the wavelength dependance of $r^{\prime}$. 
This turns out to be very substantial for cases of practical interest and so I is correctly imaged at the exit slit for only the one wavelength for which I and S are conjugates. All other wavelengths are out of focus. The size of the effect $c$ an be estimated using (18) and the conjugate relationship for the focussing mirror. For $r \gg f$ where $f$ is the focal length of the mirror, we find that the image distance $v$ is given by:

$$
v=f+\frac{f^{2}}{r}\left[\frac{\cos \alpha}{\cos B}\right]^{2}
$$

Aberrations in the mirror are not considered in this.

Using (18) and (19) we can arrive at a number of useful generalisations:

(i) Clearly from (19) we want the square bracket term to be small so that from this point of view positive order is strongly preferred. Using negative order $r^{\prime} c$ an change by as much as an order of magnitude for a factor of 4 change in wavelength!

(ii) The effect is worse for smaller $r$ values and finer gratings.

(iii) There is a trade-off with aperture. Defocus is less harmful for smaller apertures.

(iv) The effect is worse for high brightness dedicated synchrotron sources. This is because smaller values of $r$ are necessarily used and because in spite of the smaller $r$ values the source size limited resolution is still much better. The result is increased sensitivity to defocussing.

To see the effect for a practical case we show in fig. 4 the results of a design study carried out at Brookhaven for a PGM for the National Synchrotron Light Source. For each of the four $\theta$ values selected, a wavelength near the center of the range was chosen as a wavelength of exact focus. 
Since no other aberrations were considered, the resolution at these four wavelengths was equal to the source size limit represented by the straight lines in the figure. We can see from the figure that the degradation due to defocussing at all other wavelengths would be quite unacceptable.

It is of interest to consider what fig. 4 would look like for the case of, say, the DESY synchrotron. For this case, we would have about a factor of four larger $\Delta \lambda_{s}$ and with the larger I value the problem would look more managable. For NINA, the same applies. For DORIS, which has a smaller source, one would expect the problem to be correspondingly more serious. Dietrich and Kunz were, of course, aware of the defocussing problem and discussed it in their 1972 paper. However, they point out that in their case the focal shifts of the image at the slit were small enough to be compensated by a $\pm 30 \mathrm{~mm}$ adjustment of the slit position.

With these understandings, we see that the improvements in the technology of synchrotron sources have mandated a new approach to the design of PGMs. We can no longer follow the existing designs without serious loss of performance.

NEW OPTICAL ARRANGEMENTS

The fundamental problem of defocussing can only be resolved by the use of a collimator. We then have $r=\infty$ in (18) and (19) so that $r^{\prime}=\infty$ and $v=f$ and the focussing system is no longer wavelength dependent. It is easy to see that our collimator should be an off-axis paraboloid with a sufficiently grazing angle to pass the shortest wavelengths of interest and sufficient aperture not to unduly limit the system at long wavelengths. These contradicting requirements lead to a considerable problem of optical fabrication. However, assuming we wish to minimize the number of reflections by doing our reflection filtering with the grating and focussing mirror, we now have a more less defined three reflection system, consisting of the traditional components of a spectrometer, namely a collimator, disperser and focussing device. 
If we need a horizontal output bean then we wist arrange that the dejlections at the three optics add to zero. Let us suppose that the four different reflection angles shown earlier are used and also suppose that we want the best focussing mirrors for parallel light, namely paraboloids of revolution. Then we conclude that we need five off-axis paraboloids! The alternative would be to use an instrument like the 'Flipper' with collimated light. This involves only two paraboloids but the price is an extra reflection. There is obviously a good deal of judgement involved in deciding which system is best in particular cases, but it is clear that we cannot avoid addressing the problem of manufacturing off-axis paraboloids.

Our requirement is for a mirror formed by rotating a segment of the parabola $y^{2}=4 a x$ about the $x$ axis. The segment is centered on the mirror pole which is at $x_{0}, y_{0}$ (say). For a mirror of angle of incidence $\theta$ and focal length $f$ we have:

$$
\begin{aligned}
& a=f \cos ^{2} \theta \\
& x_{0}=a \tan ^{2} \theta \\
& y_{0}=f \sin 2 \theta
\end{aligned}
$$

The value of yo essentially determines how difficult it will be to make the mirror and we can see immediately that any collimating mirror we design will be difficult because of its long focal length. In fact, the difficulties are so severe that the only realistic approach is to use the new technology of diamond turning 22-25. This involves using a numerically programed lathe with a diamond tool which is interferometrically controlled. The programability means that all shapes are equally easy to figure and it is possible to simultaneously turn as many mirrors as will fit around the fixture. This latter feature is attractive for situations where the set-up costs can be amortized over several instruments. 
The technology of diamond turning has become very refined in a short time and it is now possible to work with fairly well tried materials. The combination of 6061-T6 Aluminium alloy plated with a layer of electrodeless nickel has been used successfully26,27. The electrodeless nickel layer is necessary because it is the only material which can be both diamond turned and optically polished. The optical polish is needed because the diamond turned surface; although very accurate, has the periodic pattern of tool marks left by the diamond. These must be removed by a light cosmetic polishing process 28,29 which is designed not to alter the figure.

Diamond turned optics quite similar in principle to those needed for a PGM have been produced to a considerable extent already for X-ray telescopes and microscopes30-36. They are all based on an idea originally introduced by Wolter 37,38 to improve the imaging of the grazing incidence reflecting telescope.

WOLTER TYPE OPTICAL INSTRUMENTS

The problem addressed by Wolter arose because for good off-axis imaging for any optical instrument it is necessary to closely satisfy the Abbe sine condition 39,40 . This is normally stated as the condition that an axially symetric system which is free of spherical aberration should also be free of coma, i.e., be 'aplanatic.' The condition in this case is:

$$
\frac{\sin U}{u}=\frac{\sin U^{\prime}}{u^{\top}}
$$

where $U$ and $U^{\prime}$ are the convergence angles in object and image space and $u$ and $u^{\prime}$ are the equivalent angles in the paraxial region. This is shown in fig. 5 . The condition is equivalent to saying that the 'principle surface', which is the locus of intersections of the ingoing and outgoing rays, should be a. sphere. 
The sphere intersects the aris at a point which divides $O I$ in a ratio equal to the paraxial magnification, i.e., $r^{\prime} / r=m$ (see fig. 5). The object and image are harmonic conjugates with respect to the diameter of this circle winich is thus of radius $r I^{\prime} / r-r^{\prime}$.

The effect of not satisfying the sine condition has been elucidated in detail by Conrady4l, but one can see easily that all single grazing incidence reflectors violate the condition in a fundamental way since in this case the principle surface is the reflector itself. Wolter recognized that the only way for a grazing incidence reflecting instrument to approach satisfying the sine condition was to have a second reflection. His first proposal, the socalled Wolter I telescope, is shown schematically in figure 6 . This configuration of conic sections approximately satisfies the sine condition and by means of 'aspherizing' it a little $\mathrm{e}^{38}$, it can be made to exactly satisfy the condition. Its performance as an imaging device, while poor by any normal optical standards, brought about a considerable improvement in X-ray telescopes which had previously been based on the much cruder Kirkpatrick-Baez 42 design. The technology of Wolter telescopes depends very largely on diamond turning.

It requires only a small step from the scheme in fig. 6 to conceive a microscope based on the same ideas. One simply replaces the parabola by an ellipse and the result is point to point imaging. Microscopes with magnifications up to about 10X have been designed and used 33-36 and typically they can resolve structure of about $1 \mu$ width over a field of about $500 \mu$ with an object distance of $300 \mathrm{~mm}$ and grazing angles of $1^{\circ}$. 
Designers of these instuments aiways reject the obvious variation of the ellipse/nyperbola design: namely the parabola/parabola. This has one less degree of freedom in the design equations with no compensating advantages from the point of view of designing microscopes. However, from the point of view of designing spectrometers (grating or crystal), it has the great advantage that between the two reflections at the parabolas the radiation is parallel.

\section{A PGM WITH WOLTER DERIVED OPTICS}

We now return to the PGM operating with a collimating mirror. This is shown schematically in figure 1. We see that the only effect of the plane grating at zero order is a lateral inversion, i.e., the top and bottom rays are interchanged. This is equivalent to inverting the mirror and if we imagine the two parabola system that would result from doing that we see it is very similar to a parabola/parabola, Wolter I microscope. The main differences are:

(i) It works like a microscope in reverse with magnification about 0.1 .

(ii) The object distance is much larger. From (7) it needs to be about 10m. for the Brookhaven PGM.

(iii) The two parabolas no longer necessarily have the same axis of symetry (See fig. 7).

(iv) Taking the Brookhaven vov ring as a typical dedicated synchrotron radiation source, the required field of view is about $200 \times 1000 \mu 2$ or about $4 \times 20$ arc seconds 2 : much smaller than any existing microscopes or telescopes.

(v) The optics are no longer complete circles. 
Our system is thus a generalization of the lolter I microscope working in reverse. Clearly a great deal of work would be desirable in order to understand the optics of our non-axisymetric configuration as well as the symetric one is understood. However, until that is done we can only look to certain general statements which have been established for conventional Wolter systems.

1. Exact ray-tracing is an appropriate way to predict performance.

2. Parabola/hyperbola telescopes and ellipse/hyperbola microscopes have perfect imaging for the axial object point. Adjustments to the figure to improve the off-axis imaging, e.g., to exactly satisfy the Sine Condition, can only achieve this at the expense of the perfect axial imaging 38 . In view of our extremely small field angles, we are probably better off with the exact conic sections.

3. Aberrations scale with magnification 33 so our low magnification is favorable.

4. Aberrations scale with mirror length 33 . So if we are working in negative order (beam compressed by the grating) then zero order is the worst case for aberrations.

5. Manufacturing tolerances using diamond turned optics are such that blur circles in the image plane of less than an arc second can be obtained on telescopes with much larger collection apertures and field angles than we propose. The same is true of microscopes except the collection aperture is smaller. In fact, our requirement (for the. Brookhaven PGM) for a 2 arc second blur circle capability over a $4 \times 20$ arc second 2 field of view with a collection area. $\leq 4 \mathrm{~cm}^{2}$ is quite modest. 
The ideas discussed above have been incorporated into a design 43 for

use at the Brookhaven VUV storage ring. Two units are under manufacture at time of writing. Details of the optical parameters are shown in Table II. The geometrical optical properties of this configuration have been explored by exact ray trace 44 and it has been shown that aberrations cause a negligible broadening of the geometrical image. The operating principles are illustrated in fig. 8. A $2^{\circ}$ grazing angle collimating mirror is used at $10 \mathrm{~m}$ distance from the source collecting 4mr of radiation. The collimaced light is then incidcnt on the grating also at $2^{\circ}$ grazing angle for the zero order position of the most grazing range. The focussing mirror for the latter working range thus has a $4^{\circ}$ grazing angle to restore the light to the horizontal direction. There are two gratings which $c$ an be interchanged manually using a linear translator. Wavelength scan is by simple rotation of the grating about its center. Normally, the gratings would both be 600 l/m lamina groove gratings with different groove depths ( $h$ ) for optimum blaze properties. The variation of the wavelength of maximum efficiency $\left(\lambda_{m}\right)$ with grazing angle for three different groove depths is shown in fig. 3. There are four focussing mirrors, each with a different grazing angle, and these are interchanged using a second identical linear translator. The platform shown in fig. 8 rolls along rails and each mirror is prealigned at its operating position and angle. In order to keep the number of reflections down to three, a specially designed adjustable slit mechanism is used which allows the exit slit to be placed inside an experimental chamber close to the sample position. The whole system is engineered to UHV standards. Fig. 9 shows the general layout and fig. 10 the source size limited resolution. It is difficult to calculate realistic numbers for throughput, but if we make the assumption that the storage ring source achieves its theoretical brightness and assume clean gold reflecting surfaces, then we can arrive at a sort of ideal monochromator performance and this is shown in fig. 11 . 
Clearly, these theoretical performance figures are very promising and it will be of great interest to see how far they are realized in practice. ilost of the system uses quite simple and well established techniques. The only major new element involving some uncertainty is the use of diamond turned off-axis paraboloids. The effectiveness of these has been confirmed experimentally only in situations slightly different to the present one. However, there is no reason to expect any serious problems and it is probable that no other optical fabrication method could lead to a full exploitation of the possibilities of the high brightness source. 
1. H. A. Rowl and: Phil. Mag. (4), 13, 469 (1882).

2. H. A. Rowl and: Nature 26, 211, (1882).

3. H. A. Rowl and: Am. J. Sci. (3), 26, (1883).

4. See for Example: M. Born and E. Wolf: Principles of Optics, Pergamon, Oxford (1965).

5. W. G. Fastie: J.0.S.A. 42, 641 (1952).

6. M. Czerny and A. F. Turner: 2. Physik, 61, 590, (1930).

7. M. R. Howells: Proc. Conf. on 'Synchrotron Radiation Instrumentation', Gaithersburg, Md. (1979).

8. J. A. Samson: Techniques of VUV Spectroscopy; Wiley, New York, (1967).

9. K. P. Miyake, et al: Sci. Light 18, 1, 39. (1969).

10. J. B. West, et al: J. Phys. E. 7, 137 (1974).

11. M. R. Howel1s, et al: J. Phys. E. 11,199 (1978).

12. A. H. Compton and S. K. Allison: X-rays in Theory and Experiment, $V$ an Nostrand, Princeton (1935).

13. H. J. Hageman, et a1: DESY SR-7414 (1974)

14. G. P. Williams and M. R. Howells: Brookhaven Lab. Report No 26121.

15. H. Dietrich and C. Kunz: Rev. Sci. Inst. 43, 434 (1972).

16. W. Eberhardt, et al: Nucl. Inst. Meth. 152, 81 (1978).

17. M. V. R. K. Murty: J.D.S.A. 52, 7, 768 (1962).

18. A.H.C.P. Gillieson: J. Sci. Inst. 26, 335 (1949).

19. D. J. Schroeder: J.0.S.A. $60,8,1022$.

20. H. G. Bentler: J.o.S.A. 35,311 , (1945).

21. T. Namioka: J.o.S.A. 49, 446 (1959).

22. T.T. Saito: Appl. Opt. 14, 1773 (1975). 
23. S.P.I.E. Proc. 159,2 et seg. (1978).

24. Opt. Eng. 17, 6, 569 et seg. (1978).

25. S.P.I.E. Proc. 184, 2 et seg. (1979).

26. J. Trumper, et al: S.P.I.E. Proc. 184, 12 (1979).

27. A. Aschenbach, et al: S.P.I.E. Proc. 184, 148 (1979).

28 P.C. Baker and N. J. Brown: Opt. Eng. 17, 6, 595 (1978).

29. R. E. Parks: Proc. O.S.A. Workshop on Optical Fabrication, Tucson (1979).

30. J. D. Mangus and J. M. Underwood: Appl. Opt. $8,1,95$ (1969).

31. R. C. Chase and I. P. Van Speybroeck: Appl. Opt. 12, 5, 1042 (1973).

32. W. Werner: Appl. Opt. 16, 3, 764 (1977).

33. W. H. Angustyn: Perkin Elmer Report 12541 (1975).

34. R. C. Chase and J. K. Silk: Appl Opt. 14, 9, 2097 (1975).

35. M. J. Boyle and H. G. Ahlstrom: Rev. Sci. Inst. 49, 6, 746 (1978).

36. J. K. Silk: S.P.I.E. Proc. 184,40 (1979).

37. H. Wolter: Ann. Physik 10, 94 (1952).

38. H. Wolter: Ann. Physik 10, 284 (1952).

39. E. Abbe: Sitzungs Berichte der Jenger Gesellschaft fur Medizin und Naturwissenschaft, 129 (1879).

40. W. T. Welford: Prog. in Opt. 8,269 (1976).

41. A. E. Conrady: Applied Optics and Optical Design, Part I Oxford University Press (1929).

42. P. Kirkpatrick and A.V. Baez; J.0.S.A. 38, 766 (1948).

43. M. R. Howells: Brookhaven Lab. Report No. 26858.

44. ACCOS V Program, Scientific Calculations, Inc., Rochester, N.Y. 
TABLE I

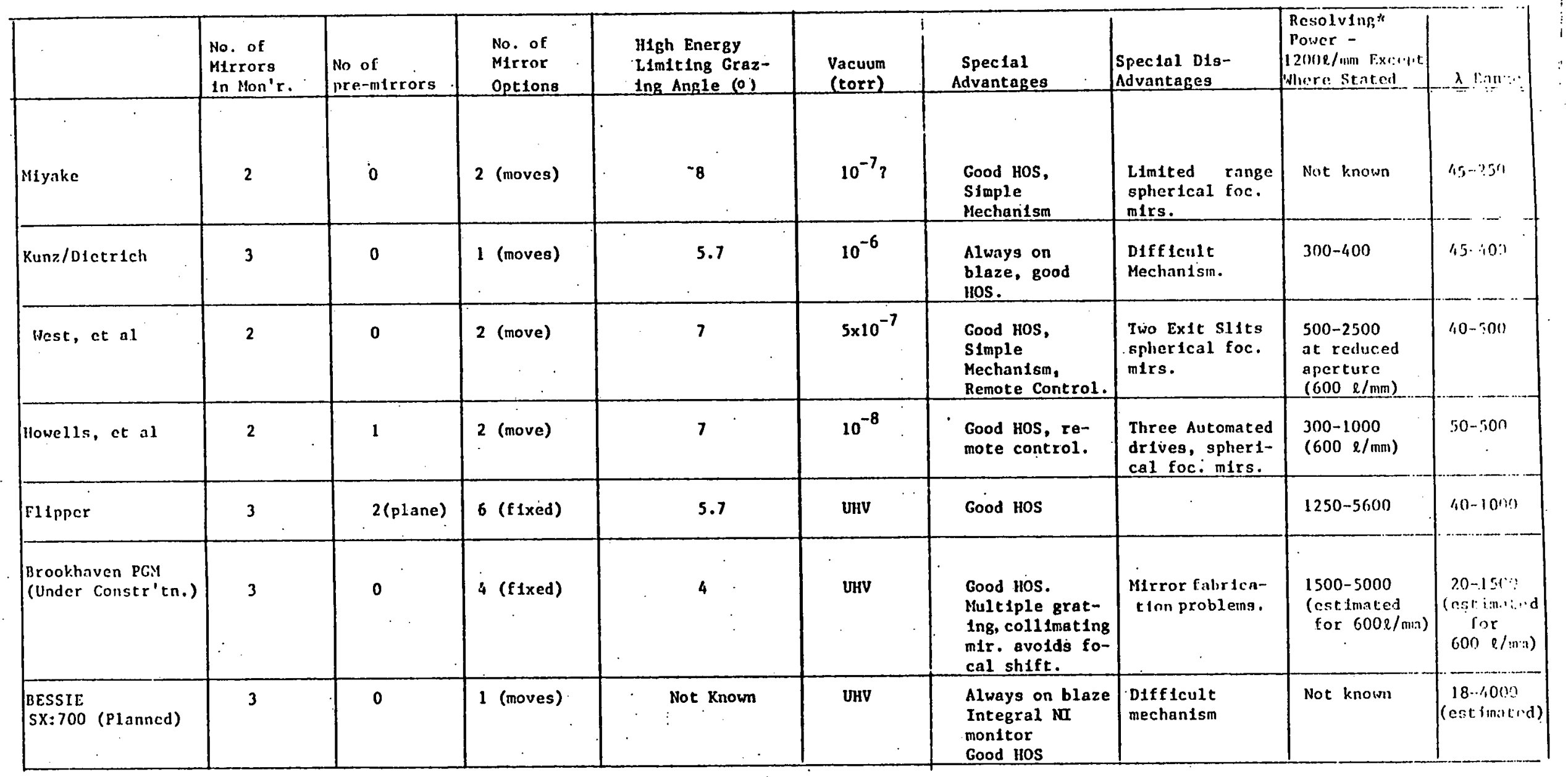

* Lower Figure Corresponds to Lowest Wavelength.

HOS - Hgh Order Suppression

$N I$ = Normal Incidence. 
The values given in this Table refer to a $600 \mathrm{l} / \mathrm{mm}$, grating of ruled width $50 \mathrm{~mm}$, used in negative order. The radiation is collimated as described in the text by a mirror with $2^{\circ}$ grazing angle and $40 \times 10 \mathrm{~mm}^{2}$ acceptance aperture placed $10 \mathrm{~m}$ from the source. All optics are presuned gold coated.

\begin{tabular}{|c|c|c|c|c|}
\hline RANGE & 1 & 2 & 3 & 4 \\
\hline $\begin{array}{l}\text { Incidence angle at } \\
\text { grating zero order (deg.) }\end{array}$ & 88 & 86 & 80 & 67 \\
\hline Mirror Slit/distance ( $\mathrm{mm})$ & 1000.0 & 1084.4 & 1170.4 & 1219.7 \\
\hline $\begin{array}{l}\text { Range of 2nd order re- } \\
\text { flection filtering( }(\AA)\end{array}$ & $20-35$ & $30-90$ & $75-160$ & $150-350$ \\
\hline $\begin{array}{l}\text { Range of } 3 r d \text { order re- } \\
\text { flection filtering. ( } \AA \text { ) }\end{array}$ & $20-40 *$ & $30-150$ & $75-315$ & $150-600$ \\
\hline $\begin{array}{l}\lambda_{m} \text { for grating groove } \\
\text { depth } 110 \AA \text { ( } \AA)\end{array}$ & 32 & 46 & 95 & 180 \\
\hline $\begin{array}{l}\lambda_{\text {m for grating groove }} \\
\text { depth } 200 \AA(\AA)\end{array}$ & $-*$ & 92 & 181 & $\underline{350}$ \\
\hline $\begin{array}{l}\text { Dispersion at the under- } \\
\text { lined } \lambda_{\mathrm{m}} \text { value }(\AA / \mathrm{mm})\end{array}$ & 0.12 & 0.77 & 2.03 & 5.00 \\
\hline $\begin{array}{l}\Delta \lambda_{\text {s }} \text { source linited at } \\
\text { underlined } \lambda_{\mathrm{m}} \text { value }(\AA)\end{array}$ & 0.017 & 0.024 & 0.055 & 0.11 \\
\hline $\begin{array}{l}\text { Incidence angle at fo- } \\
\text { cussing mirror (deg.) }\end{array}$ & 86 & 84 & 78 & 65 \\
\hline
\end{tabular}

* Note that the horizon wavelength is $40 \AA$, which sets an upper limit in these cases. 
1. Basic layout of a PGM with notation for opticai anaiysis.

2. Notation for diffraction grating equation.

3. First, second and third order thresholds for diffraction by a grating $\lambda_{1}^{G}, \lambda{ }_{2}^{G}$ and $\lambda G_{3}$ ) and reflection by a mirror $\left(\lambda_{1}^{M}, \lambda_{2}^{M}\right.$ and $\left.\lambda_{3}^{M}\right)$. These are shown as heavy continuous lines (grating) and dashed lines (mirror). Also shown are plots of wavelength against incidence angle (dotted) for various values of $\theta\left(88^{\circ}\right.$, $86^{\circ}, 80^{\circ}$ and $\left.67^{\circ}\right)$. Plots of $\lambda_{m}$ the wavelength of maximum efficiency of the $600 \mathrm{l} / \mathrm{mm}$ lamina grating for various values of the groove depth ( $h$ ) are also shown as lighter continuous lines.

4. Bandpass as a function of wavelength for various $\theta$ values with finite source distance. The straight lines show the ideal (source size limited) bandpass figures and the curves show the effect of a source at $12.5 \mathrm{~m}$ instead of infinity. The curves and straight lines show the bandpass as being the same for one wavelength for each value. This is the wavelength for which the focussing mirror was presumed to be in focus. A $600 \mathrm{l} / \mathrm{mm}$ grating, $50 \mathrm{~mm}$ wide, working in positive order is assumed. The aperture is taken to be such as to fully illuminate the grating.

5. Illustration of the Abbe Sine Condition: See Text.

6. The principle of the Wolter I telescope utilizing a paraboloid and hyperboloid of revolution.

7. Layout of the PGM with collimating mirror. The crosshatched optics are the normal configuration. Light from the source is rendered parallel by the collimating mirror, diffracted by the grating and (still parallel) is incident on the focussing mirror which focusses it to the exit slit. From an imaging point of view the grating merely interchanges the top (dashed) and bottom (chain dotted) rays. If we imagine that the mirror is positioned to receive the rays that way round without the intervention of the grating, then we get the arrangment shown with the dotted mirror. This configuration is quite similar to a Wolter I microscope (see text).

8. Artist's impression of the mechanism of the Brookhaven PGM.

9. General layout of Brookhaven PGM.

10. Source size limited bandpass figures for the Brookhaven PGM. The quoted figure of 200 is used for the source size.

11. Ideal output curves for the Brookhaven PGM assuming clean gold reflectors. 


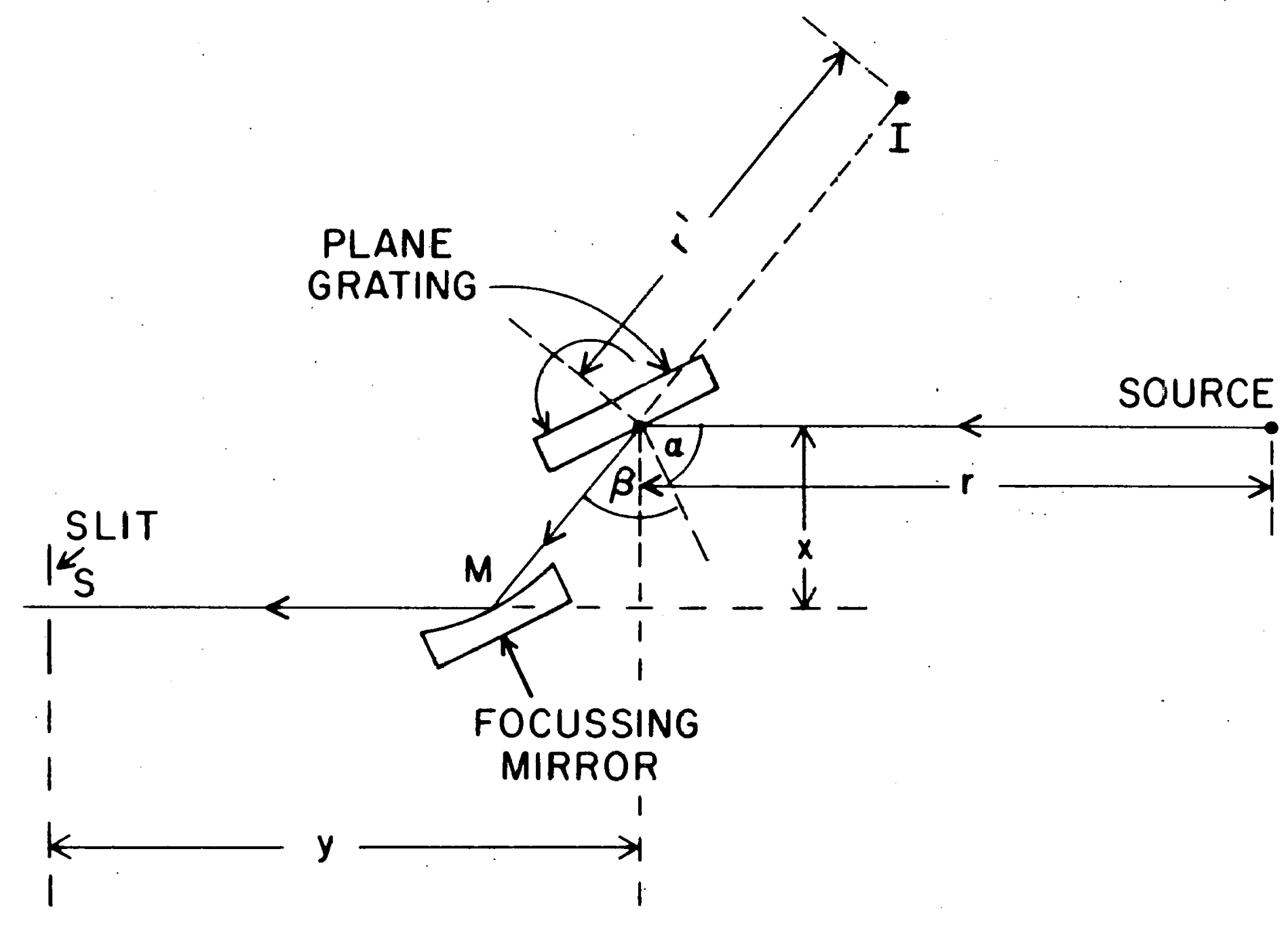




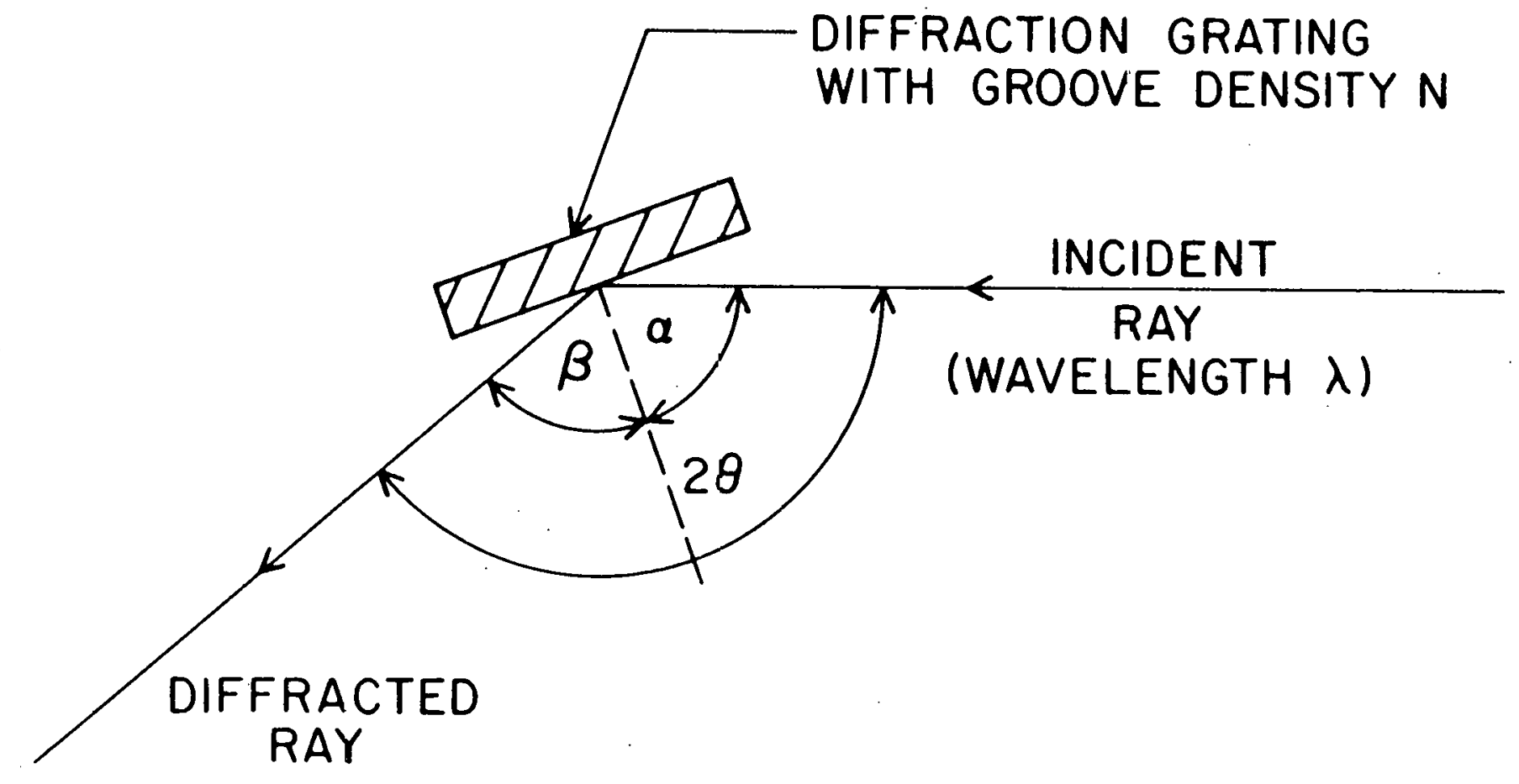




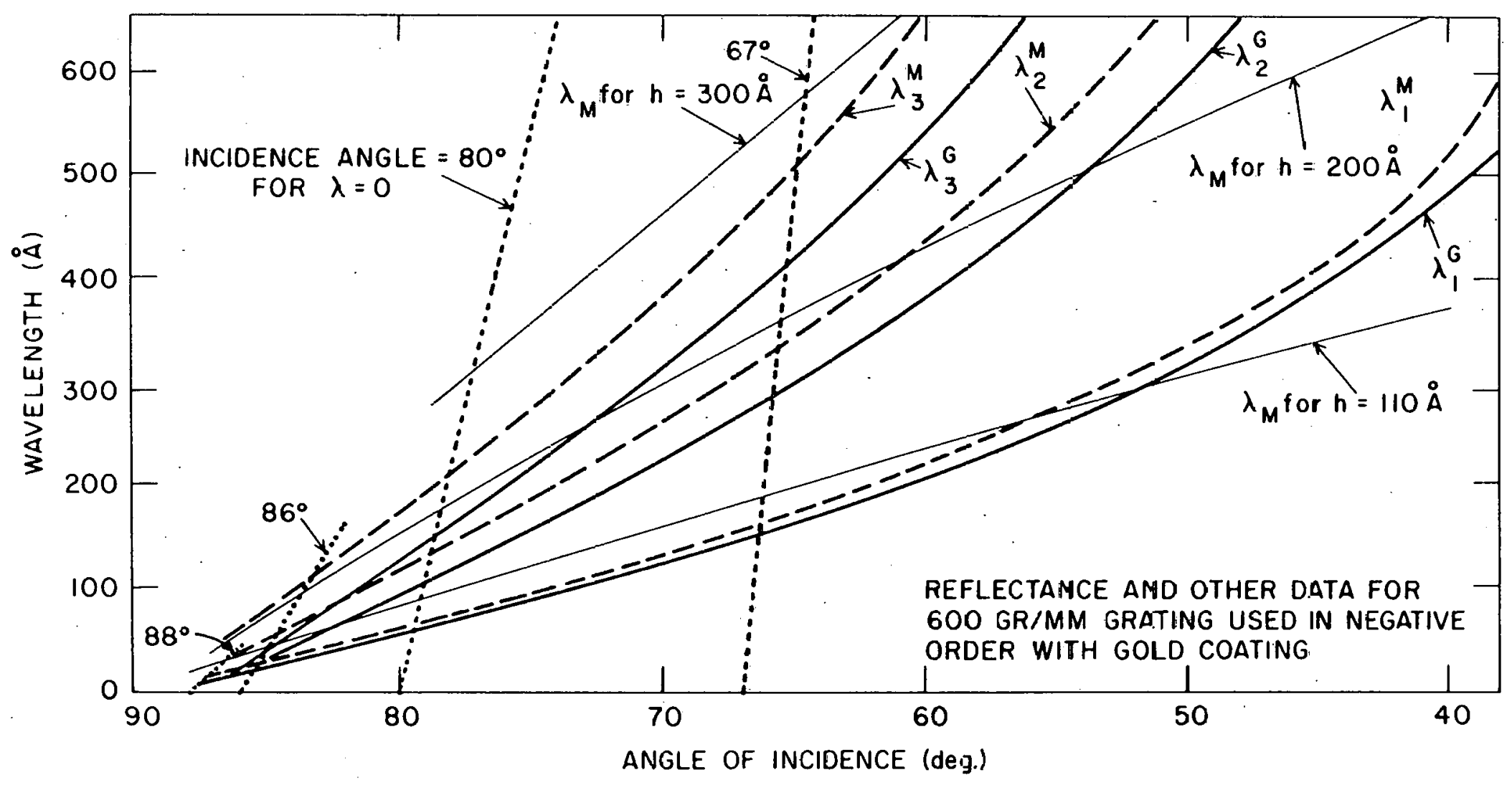




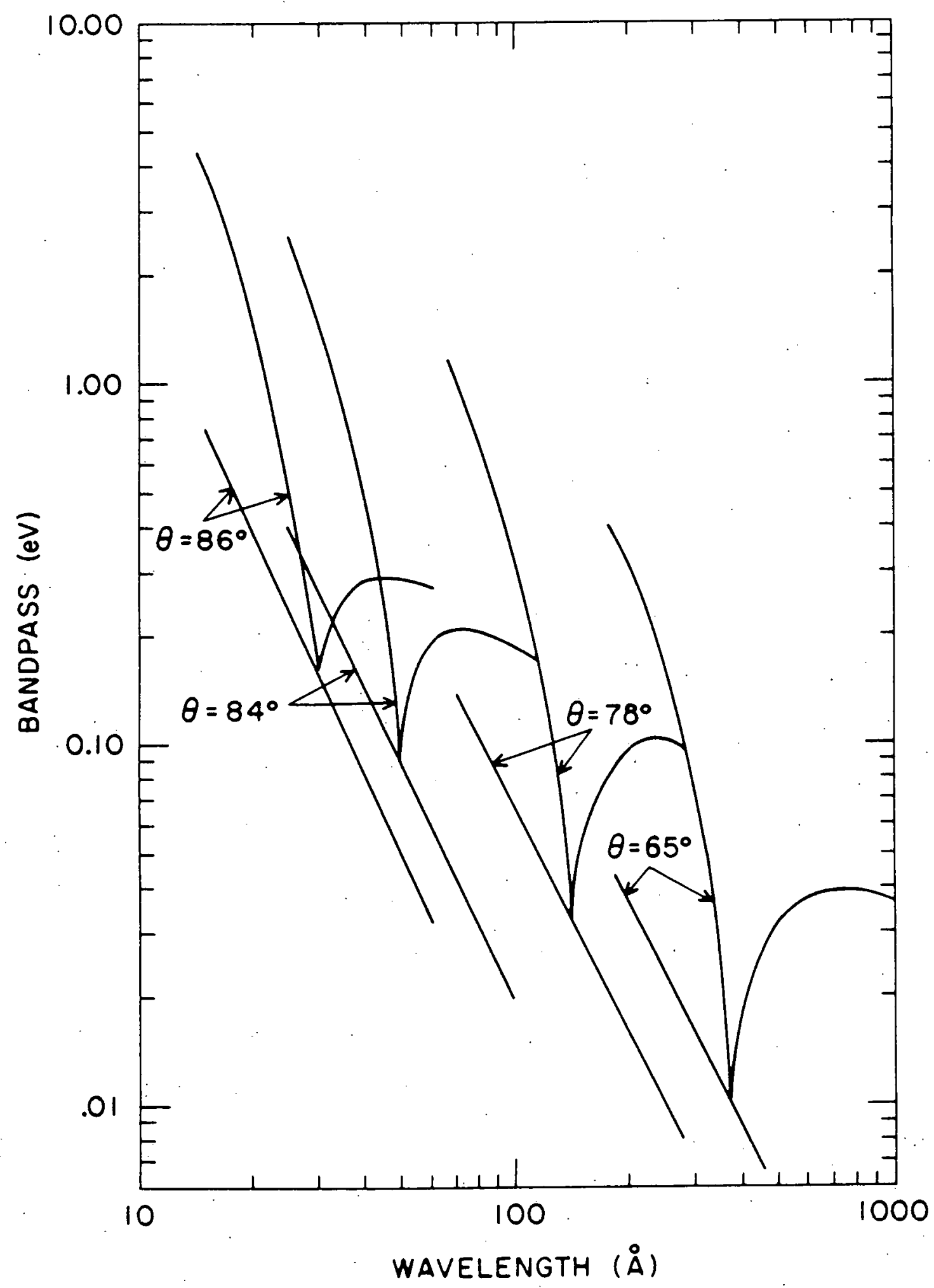




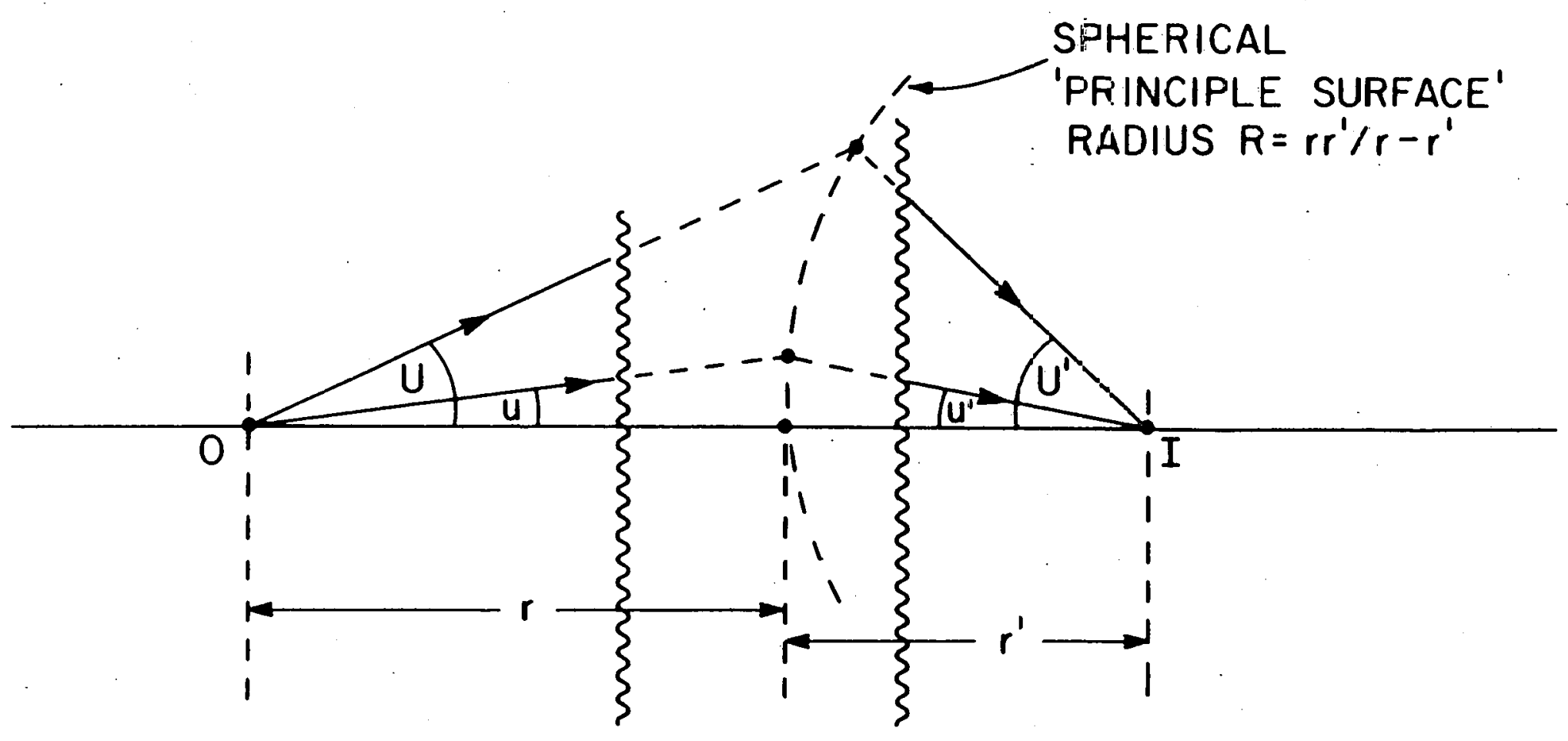




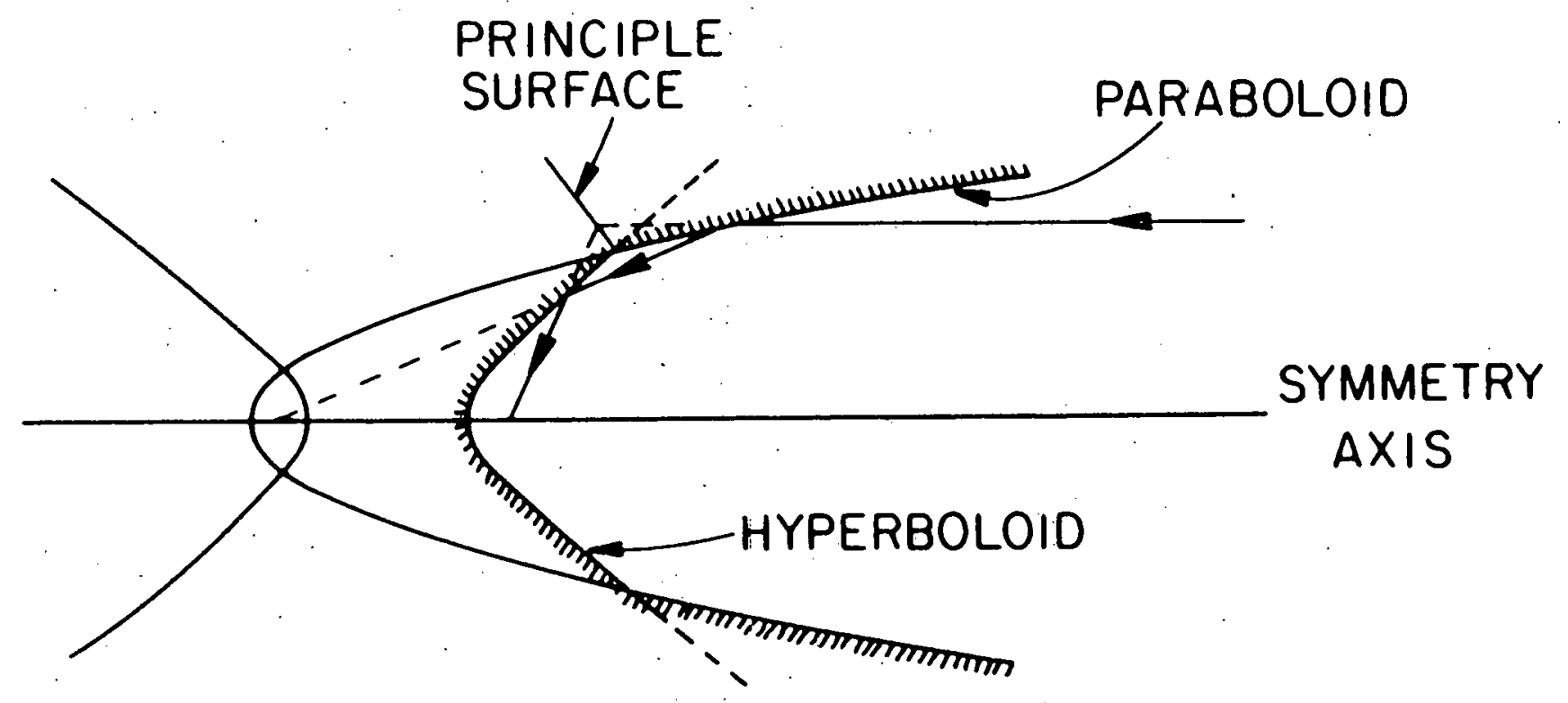




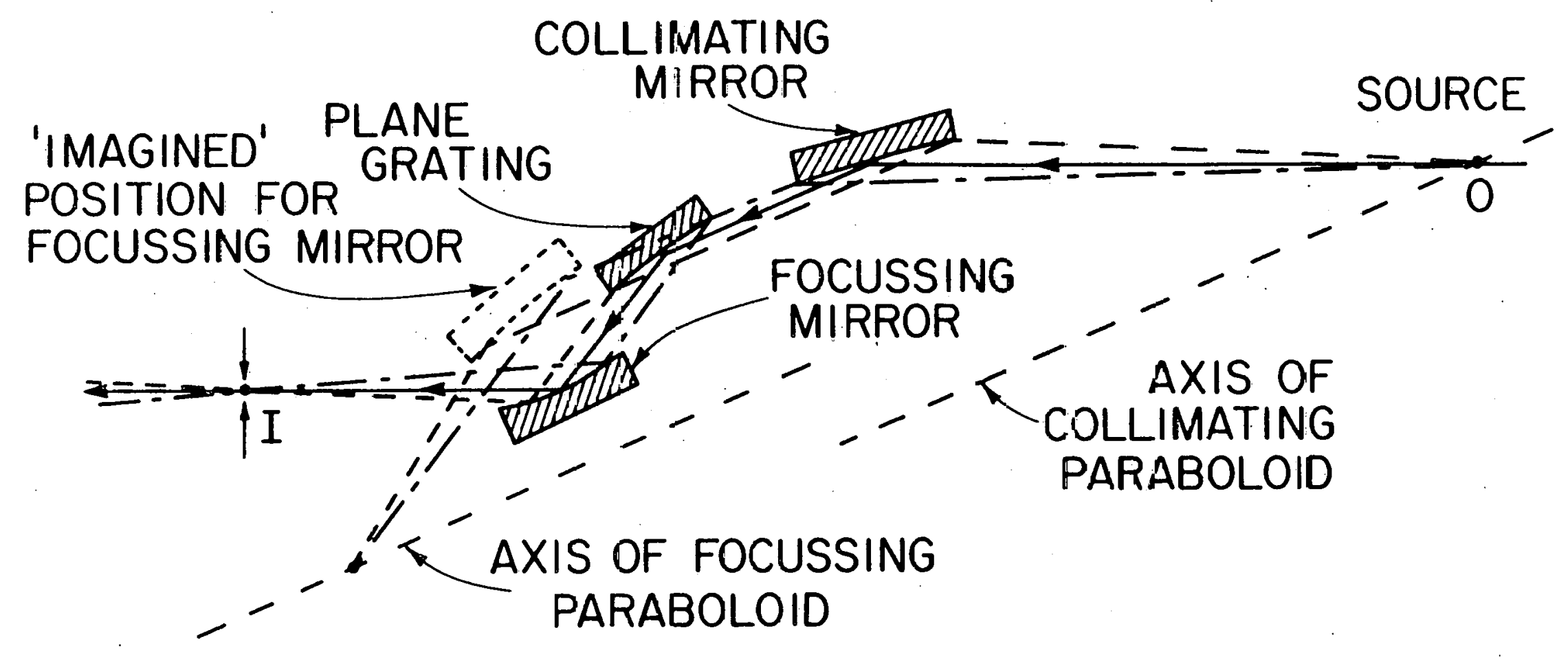




\section{GRAZING INCIDENCE PLANE GRATING MONOEHROMATOR}

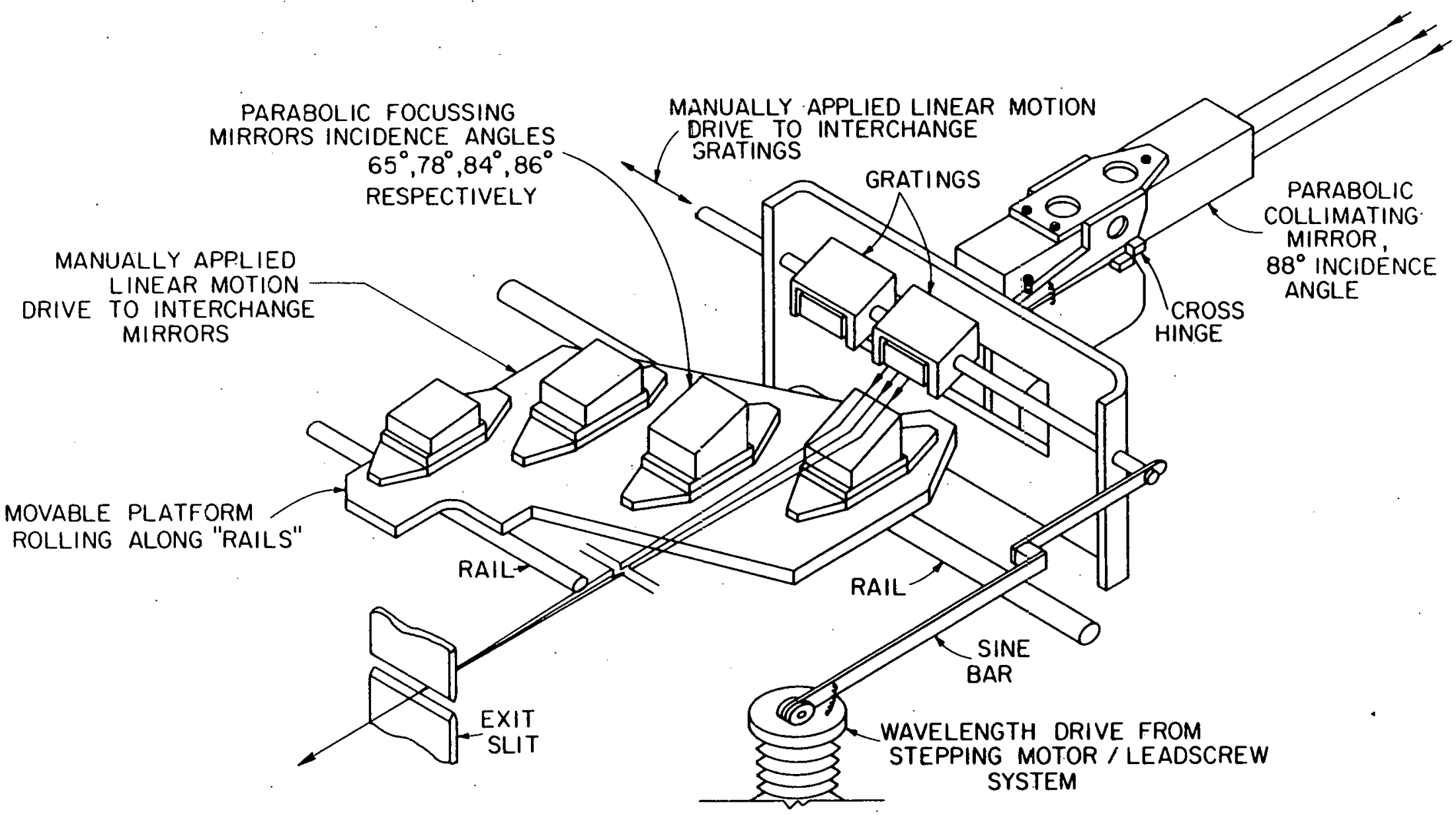



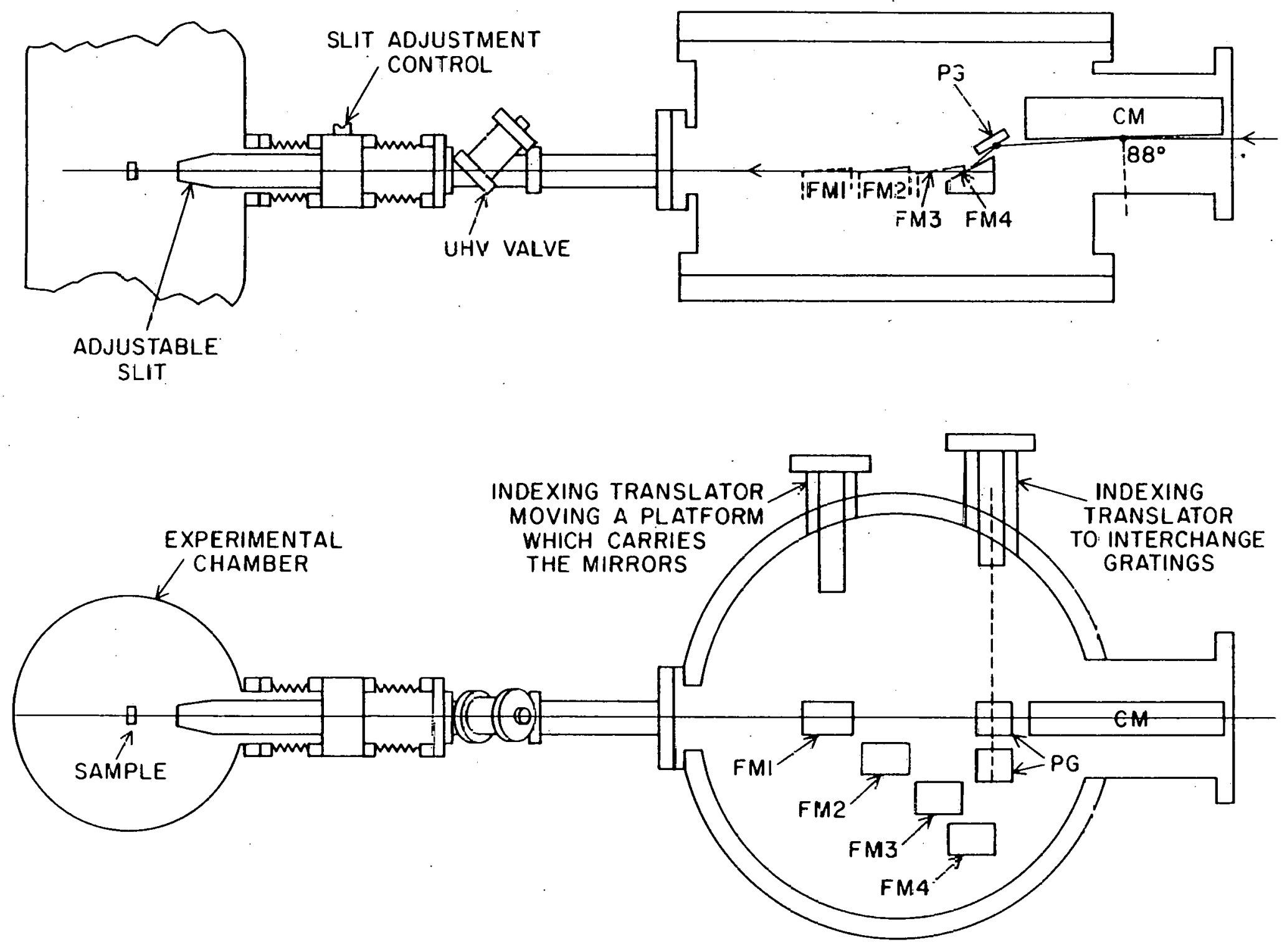


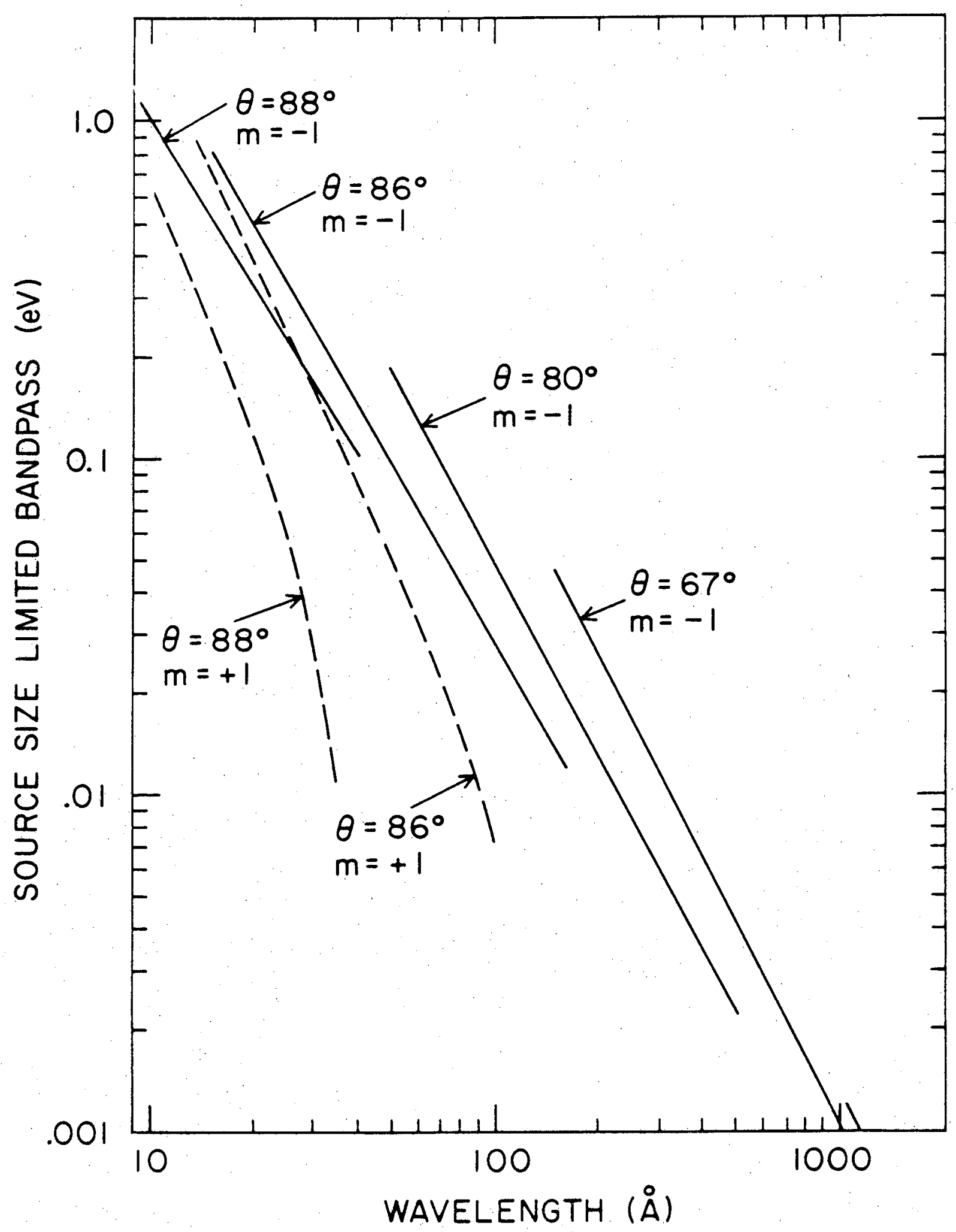




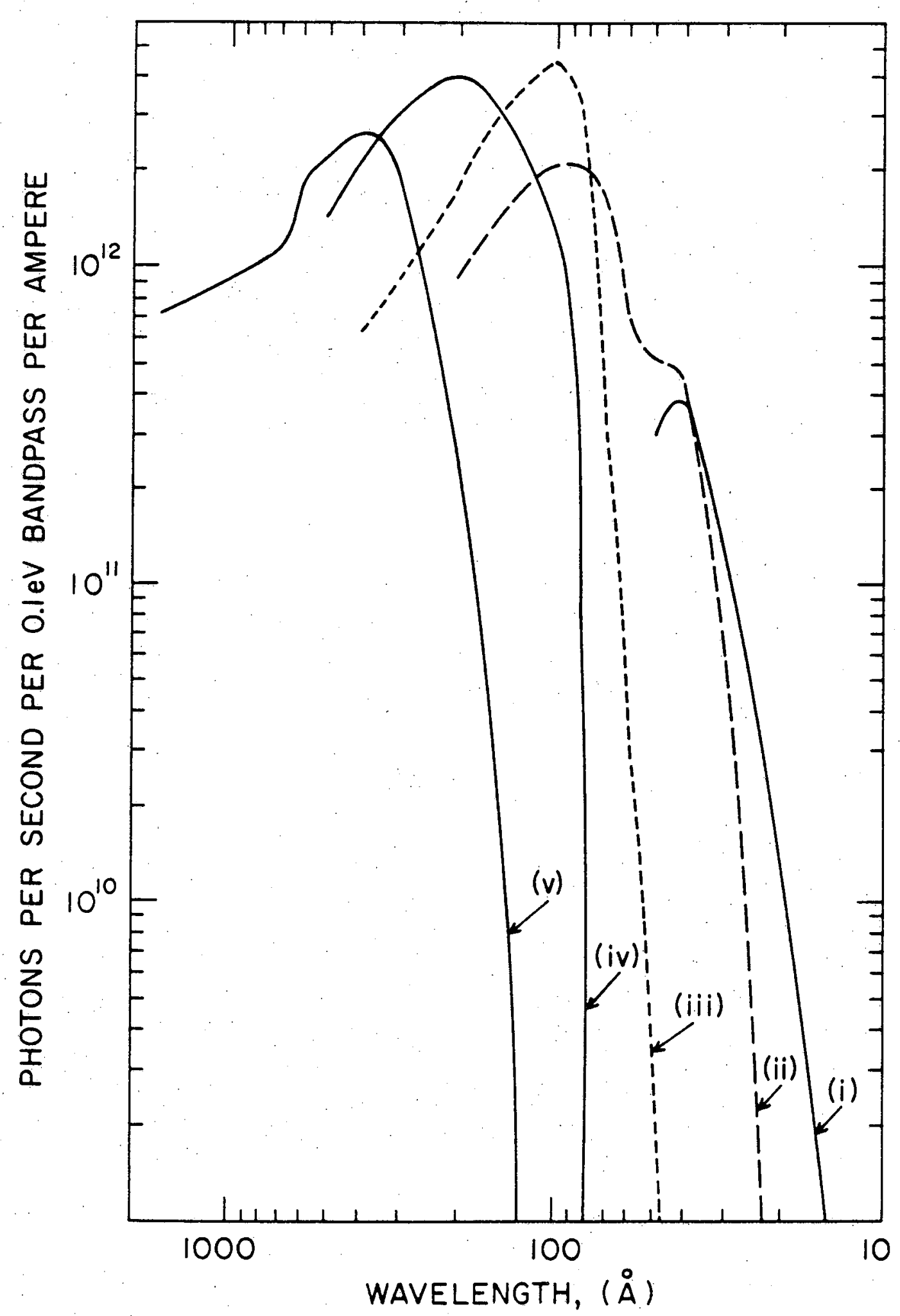

